\title{
The disconnection between tumor response and survival
}

\author{
Indraneel Mittra
}

Epithelial cancers account for more than $80 \%$ of all cancer deaths worldwide. While the rising global mortality from these cancers is largely attributable to the challenges in implementing established public health measures, it also brings into focus the unfulfilled promise of cancer treatment. This is particularly the case for the treatment of epithelial cancers with systemic chemotherapy. Extensive clinical experience has shown that while chemotherapy of advanced epithelial cancers frequently leads to dramatic tumor responses, these do not translate into commensurate improvement in patient survival. This realization has led to a search for newer systemic therapies, including biological and targeted therapies, without a critical appraisal of the reasons underlying the failure of potent cytotoxic agents to cure these cancers. The disconnection between significant downstaging and subsequent patient survival is best exemplified by the lack of benefit from myeloablative chemotherapy in metastatic breast cancer, ${ }^{1}$ and begs for an explanation that might also be universally illuminating. The well-established Goldie and Coldman hypothesis posits that the failure of chemotherapy to eradicate cancer is because of the persistence of a few mutated chemo-resistant cancer cells. More recently, residual stem cells have been implicated in resistance to chemotherapy. While these hypotheses might explain why cancer may not be cured, they do not explain why the relatively small number of cancer cells that remain following myeloablative chemotherapy do not result in significantly longer survival compared with the very large tumor cell numbers remaining after conventional chemotherapy. The Gompertzian model of tumor growth kinetics refined by Norton and Simon argues that the smaller the residual tumor volume following chemotherapy the more rapid is its regrowth rate, and unless cancer cells are completely eradicated a cure is not possible. ${ }^{2}$ Could there be another explanation?
The therapeutic approach that

treats solid cancers most effectively

is timely

surgery... $50-80 \%$ of early

breast cancers

are cured by

surgery alone

I Mittra is an Advisory Board member of

Nature Clinical

Practice Oncology.

\section{Competing interests}

The author declared he has no competing interests.

www.nature.com/clinicalpractice doi:10.1038/ncponc0772
The therapeutic approach that treats solid cancers most effectively is timely surgery. For example, $50-80 \%$ of early breast cancers are cured by surgery alone with or without systemic therapy. The fundamental difference between surgery and chemotherapy is that surgical resection destroys cancer 'outside' the body, while chemotherapy attempts to destroy cancer cells 'inside' the body. There is a large body of evidence that DNA derived from normal apoptotic cells, and apoptotic tumor cells in particular, circulates in the blood and that the level of circulating tumor-derived DNA increases dramatically after chemotherapy. ${ }^{3}$ It is conceivable that fragmented DNA released from tumor cells and carrying dominant oncogenes might initiate de novo carcinogenesis in remote targets and lead to further spread of cancer in the body. ${ }^{4}$ This notion is compatible with the recent observations that genomic profiles of solitary micro-metastatic viable cells in the bone marrow of patients with breast cancer are strikingly heterogeneous and that their genomic profiles seem substantially divergent from the cells of their corresponding primary tumors. ${ }^{5,6}$ While these findings have been interpreted to suggest that cancer cells disseminate early and evolve independently from the primary tumor, the alternative interpretation in the present context is that circulating DNA released from cancer cells is capable of entering normal cells to impart new phenotypes including neoplastic transformation. With experimental validation, such a mechanism could potentially constitute a new paradigm of cancer metastasis, in addition to the prevailing one, and support the concept of independent genesis of different metastatic clones initiated by different DNA fragments (or combinations of fragments) via a process of 'remote oncogenesis'.

Supplementary information in the form of a reference list is available on the Nature Clinical Practice Oncology website. 